\title{
Research on the Construction of Ontology- based Microcourse Resource Sharing Platform
}

\author{
Yang Feng \\ College of Informational Engineering \\ Jilin Engineering Normal University \\ Changchun China \\ yangfeng06@mails.jlu.edu.cn
}

\author{
Fan Lidan \\ College of Mechanical Engineering \\ Jilin Engineering Normal University \\ Changchun China \\ fanlidan08@mails.jlu.edu.cn
}

\begin{abstract}
E-Learning has become an important form of current education. At the same time, the development of semantic web technology provides a new way to solve the problem of information resource management, semanticbased network educational resource became an important research field in computer education technology and AI. As a main part of E-Learning, in the future, "micro course" will be a trend of education resources construction. It will provide an applicable network platform for collaborative teaching and social learning. Addressing this issue, the work presented in this paper shows the design, implementation of a new sharing platform for micro course by the advantage of ontology technology in describing and organizing knowledge. Through the three layer structure of the platform, the data layer, the logic layer and the application layer, to realize the sharing of different educational resources, provide support for the resource needs in the online learning mode.
\end{abstract}

Keywords-E-Learning; Education Sharing Platform; Micro Course; Semantic Web; Ontology

\section{INTRODUCTION}

With the further development of network technology and the unceasing emergence of application requirements, semantic web becomes a hotspot in www research field and a key technology of intelligent network services [1].The semantic web can provide more extensive application functions on the basis of the machine understanding and perform computation at the semantic level. It does not only give clearer and more completely semantic to the network information and knowledge, but also achieves that computers can understand the network information and knowledge, and the intelligentization of the network data processing and network services. Semantic web becomes a more important application in the fields as network services, agent-based distributed computing, semantic-based search engine, semantic-based digital library and so on. At the same time, with the continuous deepening of the applied research, the ontology technique as a key research of semantic web tends to be sophisticated, and there is a formation of semantic metadata describing methods, and development of semantic web begins to enter the practical application stage, such as that there is an initial exploration and application of semantic-based information representation and semantic-based searching in digital libraries and digital museums and other applications[2].
Micro course resource sharing platform is an important research field on computer education technology and AI. Constructivist learning theory holds that the four elements of the learning environment are context, collaboration, conversation, and meaning built. With the development of computer network and multimedia technology, the ways people learn changed a lot under the influence of the constructivist theory, from traditional teaching to the resource-based teaching, and then to resource-based learning [3].

With constructivist learning theory as a guide, it is an important direction of the development of human society in the 21st century digital literacy to build the sharing platform for micro courses which can select teaching content and teaching pattern according to the actual situation of students, implement individualized instruction for students. The sharing platform originated in the development of computer-aided education systems, its main feature is that according to the specific circumstances of the user to provide appropriate teaching material, in other words, it has the function of individual teaching, this feature is achieved by means of AI [4].

But, there are some problems of current micro course resource sharing platform as follows:

\section{A. Low Retrieval efficiency}

For teaching knowledge is excessive and extensive, the traditional keyword-based search technology can not meet the needs. It is necessary to use appropriate ontology to describe educational resources library and build ontologybased knowledge architecture for solving these problems.

\section{B. Weak semantic}

High semantic is necessary for communication between different educational application platforms. The reason why making the exchange and sharing of knowledge difficult is that standard of describing the teaching knowledge base is not the same all the time and the knowledge represents in different ways[5].

\section{Lack of intelligence}

A lot of current teaching systems can not provide Student Information automatically for students to do targeted studying, and make teachers unable to prepare the suitable learning content following the cognitive model of students, and guide the student to learn by their cognitive characteristics and the changes in learning. 
In response to problems of the micro course resource sharing platform construction and application needs, this paper introduced key technology of semantic to the building of education resources, presented the primary frame of micro course resource sharing platform based on semantic web technologies, started with the significant aspects of the building of micro course resource sharing platform, such as Semantic-based metadata description, education and resource library ontology model, pattern matching between the resource pool, and discussed the core concept of the framework and key technology.

\section{ONTOLOGY}

In this section the most generally accepted understanding of ontology is presented.

Since Ontology is a Conceptual Model which is used for describing information systems on the level of semantics and knowledge, it has been used in many fields. Since Ontology is not a static model, it has to have the ability to capture the changes of meanings and relations.

In research field of AI, there are many definitions of ontology. Among them, the most frequently adopted is Gruber', that is, an ontology is an explicit specification of a conceptualization [6]. An ontology $\mathrm{O}$ is a 5-tuple $\mathrm{O}=(\mathrm{C}, \mathrm{I}$ $\mathrm{R}, \mathrm{F}, \mathrm{A})$, where $\mathrm{C}$, I are concepts and instances respectively. $\mathrm{R}$ is a relation set, $\mathrm{F}$ is a function above $\mathrm{C}$, and $\mathrm{A}$ is a set of (ontological) axioms--specifying the intended interpretation of the vocabulary in some domain of discourse. Base on Gruber's definition, there are some types of ontology matching: concept to concept, property to concept, property to property, scenario and constraint and so on [6]. .

Ontology plays an important role in the construction of the educational resources library, which can achieve tripartite sharing [5]:

- Sharing of resources contents. It means that resource libraries provide resources to each other, at the same time get resources they do not have from each other.

- Sharing of technology. It means that when different organizations or individual build their own resources library, if they can follow the same standard, the same resource representation mode and the same attribute marking method, then they can share one interface.

- Sharing of concepts. It means that for achieving semantic understanding, make sure that the different objects being established, maintained and used of knowledge during this area share the same concept.

\section{SHARING PLATFORM OVERVIEW}

We designed an ontology-based sharing platform for micro course resources. It can realize the access and reuse of micro course, achieve the interoperability between the distributed repositories of micro course resources and optimize resource life-cycle management mechanism. Most importantly, it provides semantic-based search, because such a system framework provides an integrated, global-oriented concepts and ontology representation mechanisms, and the semantic-based resource formulation. The structure of the platform consists of three parts. It is shown in Fig.1. More technology details of this platform will be discussed in Part IV.

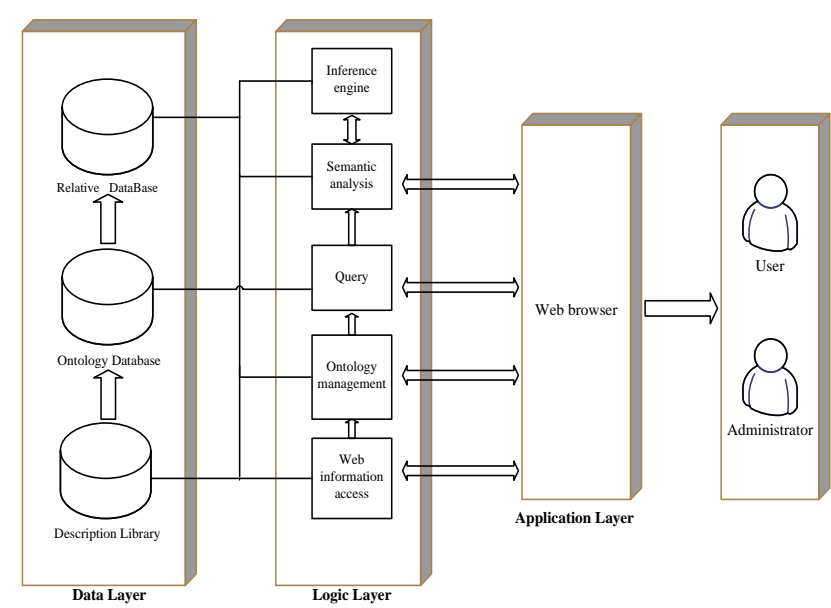

Figure 1. Ontology-based sharing platform for microcourse resourse

\section{A. Data Layer}

Data Layer is composed of three parts: ontology library, education resource Description Database and MySQL/DB2/oracle Database. As the storage medium of knowledge resource ontology, this layer is responsible for creating and refining a structural description of the OWLbased knowledge ontology, which is the direct source of knowledge retrieval. In addition, in order to achieve knowledge sharing and reuse, it will carry out semantic annotation to the relevant resources in the web page and store them in the ontology library by the data acquisition components.

\section{B. Logic Layer}

As the main application logic layer, it carries on the retrieval of system knowledge. It consists of five parts, namely, ontology management component, semantic analysis component, inference engine, query component, and Web information access component. After user's query requests are submitted by search engine, semantic analysis component obtains the concepts and semantic relations that user needs through the semantic analysis, and then Jena OWL inference engine takes on reasoning on the basis of this concepts and semantic relations, ultimately the query Beans accesses this resource ontology library and returns the query results to the user.

\section{Application Layer}

It is the interactional interface between user and system. Users access the system through a browser. The user interface is responsible for receiving queries and displaying the search results to the user. In this layer, the ontologies are shielded to user's web page, though they are adopted. So the semantic information of web page are transparent to the user. In order to achieve this separation of display and logic, JSP and Java Bean technology have been used in the development of this layer.

\section{KEY TECHNOLOGIES OF SHARING PLATFORM}

In this section the key technologies of this sharing platform are presented. It includes web services, 
processing of user request, semantic parsing and inference engine.

\section{A. Web Services}

Representation Layer may also be known as the Network Service Layer, its main task is to achieve interaction with the user. Under the case of a variety of the resource building ways, the web service technologies are selected in order to integrate distributed resource library of education effectively and share information between them [7]. On the basis of a variety of heterogeneous platforms, Web Services are available to build a common platformindependent, language-independent technical level, which applications of different platform rely on to implement the connection and integration between them [8]. Throughout the resource library, as a service provider, each local resource make their own services and features promulgate to the global system, which UDDI (Universal Description Discovery and Integration) on it. And then the UDDI is responsible for the registration and sending. In this, all the information is send to the entire system through the SOAP (Simple Object Access Protocol).

The process of searching and accessing resources through the browser is as follows:

First, the user sends a search request through a browser If the request is a keyword or content search for a local resourse, UDDI will accept it and send the results from service registry module to command forwarding module; Instead, if the search is based on the concept, it will be passed to the semantic layer, where the ontology mapping and the transformation of the request is completed. Then, the command forwarding module is positioned to provide the local repository for the service, and the Services Web is executed. Finally, the result is returned to the restructuring module, which is to complete the restructuring of the request, and sent to the user.

\section{B. Processing of user request}

In our framework, user takes on resource search by an ordinary combination method that fields are limited or natural statement. Learning objects belong to different local resources, they are geographically separated. Different resource libraries are usually built on different computer platforms, data formats and programming languages, which provide their own mechanisms to query and access resources. In this case, the central server needs a certain mechanism to orient local resource libraries that can provide server, and then user request will be sent to local servers. In the specific system design, we refer to a number of mathematical methods [8]. Fig 2. illustrates how a user request to be processed by these modules.

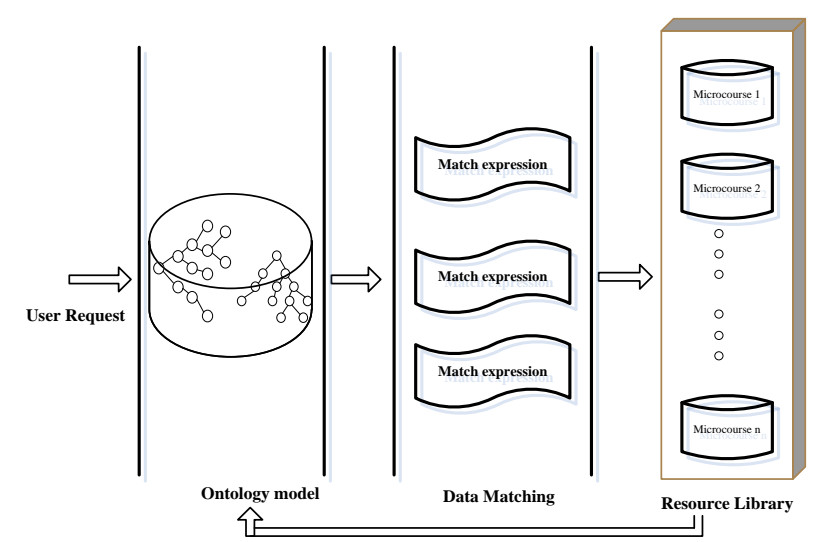

Figure 2. Processing of user request

\section{Semantic parsing}

In our framework, semantic parsing is responsible for the transition from the user requests submitted by search engine to concepts of ontology. The conversional process of user semantic to machine presentation need query ontology library, analyze user intents and use Jena Owl inference mechanism to complete. As user query are uncertainty in a certain degree, it is feasible that expand the concept set of queries according to the needs of users. Semantic expansion rule is defined as:

- If $\mathrm{O}$ is Literlas type, then $\mathrm{S}$ is the center of expansion, the concepts for a query can be obtained according to the concepts and attribute relations of $\mathrm{S}$. If $\mathrm{S}$ does not exist, the concepts are not to be taken.

- If $\mathrm{O}$ is a resource, then $\mathrm{O}$ is the center of expansion, the concepts for a query can be obtained according to the concepts and attribute relations of $\mathrm{O}$.

The query processing is the most important part of semantics retrieval. It can be described as the following RI model: ontology model and ontology-based web resources identity model. In those, web resources R and the query Q are built based on ontology o and they are relevant if and only if $\mathrm{R}$ satisfy $\mathrm{Q}$. It describes that $\mathrm{R}$ and $\mathrm{O}$ imply logically the query $\mathrm{Q}: \mathrm{O}^{\wedge} \mathrm{R}-\mathrm{Q}[9]$.

There are two proven methods that can convert the query keyword into ontology description. [10] proposed a semantic-based retrieval approach: ontology matching graph, that is, semantic correlations are brought forward between ontology, relationships and ontology graph. According to these semantic correlations, the framework for ontology graph matching that can compute these correlativities is designed. In [11], an alternative idea of semantic retrieval is given. It presents a search architecture that combines classical search techniques with spread activation techniques applied to a semantic model of a given domain. Given ontology, weights are assigned to links based on certain properties of the ontology, so that they measure the strength of the relation. Spread activation techniques are used to find related concepts in the ontology given an initial set of concepts and corresponding initial activation values[12]. These initial values are obtained from the results of classical search applied to the data associated with the concepts in the ontology. Two test cases were implemented, with very positive results. It was 
also observed that the proposed hybrid spread activation, combining the symbolic and the sub-symbolic approaches, achieved better results when compared to each of the approaches alone[13].

\section{Inference engine}

This is the critical control module of the whole framework. Through the inference and search, knowledge user' need will be obtained, at the same time, intelligent teaching methods and strategies can be gotten[14]. Inference engine is responsible for parsing and reasoning owl documents. The aim is to read ontology from general file, store in a specific model in order to facilitate process, and then carry on ontology-based semantic inference according to certain rules, which is the semantic retrieval crucial step[15]. This process uses Jena development kit to implement. As shown in Figure 3, the operational principle of inference engine is: inference engine registration mechanism creates reasoning machine based on basic RDF triples and ontology. After this process, model objects contained inference mechanism, InefreneeGnarh and InGfraph can be generated. In Jena, Garph is also known as Model and its manifestation is ModelInterafee. And then, it can take on operation and processing to this model by using the Model API and Onotlogy API, information retrieval on semantic level can be achieved consequently.

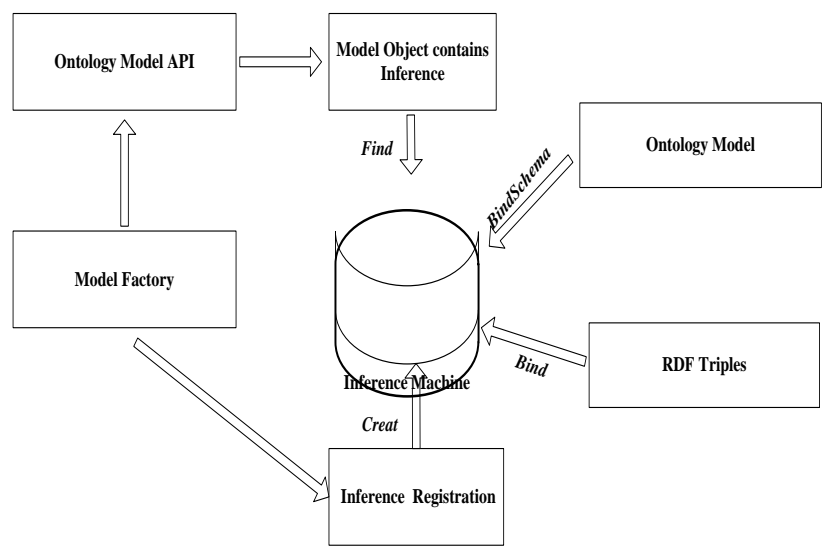

Figure 3. Operational principle of Inference Engine

\section{CONCLUSION AND FUTURE WORKS}

In this paper, we have proposed the design, implementation of a new sharing platform for microcourse resources library. By using ontology-based semantic technology, we create an open platform for new educational resources, achieve interoperation between heterogeneous resources and realize the centralized management of resources and contents distributed stored. Based on these, the resource library can become sharing, distribution, open and make interoperation between heterogeneous resource be supported.

With the rapid development of educational contents, curriculum is also changing. The production and update of education resources are more frequently. Through using ontology' standardized expression and work process and establishing education resource library based on common standard, the widespread sharing and reuse of resources can be achieved and the consensus of concept can be formed. It will be an important direction for construction and development of education resource library.

In the future work, we will extend our current work along three directions. First one is the improvement of the architecture. Second direction is to realize it with more complex ontologies when rich data sets related to our framework are available. The last is comparing with other micro course resources sharing platform.

\section{REFERENCES}

[1] Ding Y, Foo S. Ontology Research and Development Part 1 - A Review of Ontology Generation[J]. Journal of Information Science, 2012, 28(2): 123-136.

[2] Tim Berners-Lee, J.Hendler, and O.Lassila, The Semantic Web, Scientific American Vol.284, no.5, PP.34-43, 2011.

[3] Harper B, Hedberg J. Creating Motivating Interactive Learning Environments: a Constructivist View. Proceedings Australasian Society for Computers in Learning. In Tertiary Education Conference, 2010: 906-908.

[4] Geraldine Lefoe. Creating Constructivist Learning Environments on the Web: The Challenge in Higher Education. ASCILITE, 1998, 453-464.

[5] Aroyo L, Dicheva D. A IM S: Learning and teaching support for WWW - based education [J ]. International Journal for Continuing Engineering Education and Lifelong Learning, 2009, 11 (2) : 152164.

[6] Gruber T R. A Translation Approach to Portable Ontology Specifications[J]. Knowledge Acquisition, 1993, 5(2): 199-220.

[7] Yolaine Bourda. The semantic Web for learning resources [DB/OL]. http:// ieeexplore.ieee.org/ iel5/ 8621/ 27318/ 01215104.pdf.

[8] HONG-ZHE LIU, etc. An ontology- based architecture for distributed digital museums [DB/OL]. http:// ieeexplore.ieee.org/ie15/10231/32623/01526913.pdf

[9] Olivier Corby, Rose Dieng-Kuntz, Catherine Faron- Zucker. Querying the Semantic Web with the CORESE search engine. In R. LoPez de Mantaras and L.Saittaeds, Proc. of the 16th European Conference on Artificial Intelligence (ECAI' 2004), sub conference PAIS, 2004, Valencia, 2004.8

[10] Jiwei Zhong, Haiping Zhu, Jiamning Li et al. Conceptual Graph Matching for Semantic Search, 10th International Conference on Conceptual Structures, ICCS2012 Borovets, Bulgaria, Ju1y1519,2002

[11] Cristiano Roeha, Daniel Sehwabe, Mareus PoggideArago. A Hybrid Approach for Searching in the Semantic Web. WWW2004, New York, USA, 2014.5

[12] Arch-int, N., \& Arch-int, S.. "Semantic ontology mapping for interoperabilityof learning resource systems using a rule-based reasoning approach." Expert Systems with Applications, vol.40, Dec. 2013 , pp. 7428-7443.

[13] ACM. (2013). ACM Digital Library. URL: <http://dl.acm.org/>.

[14] Araujo, S., Tran, D., DeVries, A., Hidders, J., \& Schwabe, D. (2012). Serimi: Class-based disambiguation for effective instance matching over heterogeneous web data. In Z. G. Ives, \& Y. Velegrakis (Eds.), WebDB (pp. 25-30). URL: <http://dblp.unitrier. $\mathrm{de} / \mathrm{db} /$ conf/webdb/webdb2012.html\#AraujoTDHS12>.

[15] Behkamal, B., Naghibzadeh, M., \& Moghadam, R. (2010). Using pattern detection techniques and refactoring to improve the performance of ASMOV. In $5^{\text {th }}$ International symposium on telecommunications (IST) (pp. 979-984). 